\title{
Long-Term Normal Renal Function after Drastic Weight Reduction in Patients with Obesity-Related Glomerulopathy
}

\author{
Assumpta Serra $^{a} \quad$ Anna Esteve $^{b} \quad$ Maruja Navarro-Díaz $^{a} \quad$ Dolores López $^{c}$ \\ Ioana Bancu ${ }^{a}$ Ramón Romero ${ }^{a}$ \\ a Department of Nephrology, Hospital Universitari Germans Trias i Pujol, Universitat \\ Autònoma de Barcelona, Badalona, Spain; ${ }^{b}$ Department of CEESCAT, Hospital Universitari \\ Germans Trias i Pujol, Universitat Autònoma de Barcelona, Badalona, Spain; ${ }^{c}$ Department of \\ Pathology, Hospital Universitari Germans Trias i Pujol, Universitat Autònoma de Barcelona, \\ Badalona, Spain
}

Key Words
Bariatric surgery $\cdot$ Hypertension · Obesity $\cdot$ Kidney disease $\cdot$ Proteinuria

\begin{abstract}
Aims: No long-term studies of renal function evolution in morbidly obese (MO) patients after weight loss are available. The aim of our work was to ascertain the long-term influence of drastic weight reduction on renal function in $\mathrm{MO}$ patients with obesity-related glomerular lesions. Methods: $92 \mathrm{MO}$ patients with normal renal function and biopsy evidence of mild obesity-related glomerulopathy underwent bariatric surgery (BS) and subsequent drastic weight loss. A long-term prospective follow-up (mean duration: $76 \pm 42$ months) was carried out. Basal renal biopsies and basal and long-term metabolic and renal function studies were performed in all cases. Linear mixed models were applied. Results: Blood pressure dropped early after BS and remained stable thereafter. Creatinine clearance and BMI fell in the first 2 years, rose slightly after 5 years and then remained stable. Serum creatinine and albuminuria decreased throughout the follow-up period. Renal function and albuminuria evolution showed non-significant differences in relation to the number of glomerular lesions. Conclusions: Drastic weight loss in BS-treated MO patients with pre-surgical normal renal function and mild obesity-related glomerular lesions is associated with short- and long-term maintenance of normal renal function and improvement in both arterial hypertension and albuminuria.
\end{abstract}

(c) 2015 S. Karger GmbH, Freiburg 
Serra et al.: Long-Term Normal Renal Function after Drastic Weight Reduction in Patients with Obesity-Related Glomerulopathy

\section{Introduction}

Evidence of a link between obesity and kidney disease is growing [1]. Observed glomerular hyperfiltration, microalbuminuria, and proteinuria could be the earliest manifestations of renal involvement in asymptomatic obese patients [2,3]. Weight loss achieved with different types of surgical or lifestyle methods results in a short- or medium-term improvement in proteinuria and microalbuminuria in obese patients $[4,5]$. Nevertheless, the short- or medium-term (up to 24 months) effects of weight loss on the glomerular filtration rate (GFR) are variable. In obese patients with renal hyperfiltration, the greatest GFR decrease is achieved by bariatric surgery [2-4]. If this hyperfiltration improvement translates into long-term renal benefits remains to be seen. On the other hand, the short-term effects of weight reduction on GFR in obese patients with chronic kidney disease are quite variable [4, 5]; GFR remains stable in some patients [6-8], whereas there is improvement in others [9]. Therefore, knowledge of the long-term evolution of renal function and/or proteinuria/albuminuria in patients undergoing drastic weight reduction is lacking.

Several glomerular lesions, e.g., glomerulomegaly, podocyte hypertrophy, mesangial matrix increase (MMI) and focal and segmental glomerulosclerosis (FSGS), are associated with obesity. These lesions are collectively known as obesity-related glomerulopathy [1014]. The short- and long-term evolution of renal function after weight loss is less well known in patients with obesity-related glomerulopathy than in the overall group of obese patients. Although some reports exist on the spontaneous evolution of renal function in these patients $[11,15,16]$, studies dealing with weight loss influence on renal function in these patients are scarce and include only few cases with short follow-ups and small weight reductions [17-20]. Therefore, the long-term evolution of renal function and/or proteinuria/albuminuria in morbidly obese patients with obesity-related glomerulopathy undergoing drastic weight reduction is unknown.

The first aim of our observational study was to describe the long-term evolution of blood pressure, renal function, and albuminuria in a cohort of morbidly obese patients with normal renal function who were prospectively followed up after bariatric surgery and subsequent weight loss. Our second aim was to assess the possible short- and long-term influences of obesity-related glomerular lesions on the evolution of blood pressure, renal function, and albuminuria.

\section{Patients and Methods}

In a previous study [12], 95 morbidly obese patients with normal renal function were investigated. Of these, 92 were included in the present study. The patients underwent open bariatric surgery at our institution between December 2001 and November 2005. They were invited to participate in a study including complete clinical and biochemical evaluation before surgery, renal biopsy at the time of surgery, and clinical and biochemical long-term follow-up post-bariatric surgery (with at least one clinical and one biochemical evaluation after surgery). All patients met the surgery criteria established by the National Institutes of Health consensus [21]. The surgical technique performed was gastric bypass using either the method described by Fobi et al. [22] or that of Salmon [23]. Both techniques combine a permanent restriction of volume ingested (gastroplasty) with moderate or mild food malabsorption.

No patient was on insulin, lipid-lowering drugs, or oral antidiabetic treatment at the start of the study. 29 patients were being treated for arterial hypertension (7 with angiotensin-converting enzyme inhibitors (ACEIs), 3 with angiotensin II receptor blockers (ARBs), 4 with diuretics, 2 with $\beta$ blockers, and 13 with ACEIs and/or ARBs in combination with other drugs). Antihypertensive drug dosages were reduced (mainly nonACEIs and/or ARBs hypotensive drugs) during follow-up to maintain blood pressure within normal levels.

Clinical and biochemical studies were conducted prior to surgery and throughout follow-up in all patients studied. Blood pressure was measured using a standard mercury sphygmomanometer of appro- 
Serra et al.: Long-Term Normal Renal Function after Drastic Weight Reduction in Patients with Obesity-Related Glomerulopathy

priate cuff size. Preoperative (basal) and long-term follow-up blood and urine samples were drawn as previously described [12]. Serum glucose, HbA1c, total cholesterol, LDL- and HDL-cholesterol, triglycerides, serum creatinine, creatinine clearance, and 24-hour albuminuria were measured as previously described [12].

Renal biopsies were routinely processed for histological studies using standard procedures and image analysis techniques to detect the presence of glomerulomegaly [12].

In order to evaluate the possible influence of glomerular lesions on the evolution of clinical and renal function variables throughout follow-up, we selected the most common forms of obesity-related glomerular lesions [11,12,14]. Specifically, the lesions selected were MMI and/or FSGS, glomerulomegaly, and podocyte hypertrophy. MMI was considered to be present when mesangial sclerosis causing diffuse widening of the mesangium was identified, while FSGS was diagnosed when focal and segmental consolidation of the glomerular tuft by increased extracellular matrix with obliteration of capillary lumina was identified. On the other hand, the diagnosis of glomerulomegaly required the glomerular area to be above the mean glomerular area plus two standard deviations (SD) in a control group of normal-weight subjects [12] and the absence of other renal lesions, while podocyte hypertrophy was diagnosed when podocyte enlargement with large nuclei and prominent nucleoli, with or without intracytoplasmic protein resorption droplets, was present in the absence of any other renal lesion.

Our patients were classified in 3 different histological groups according to the number of lesions of obesity-related glomerulopathy presented: no lesion, 1 lesion and 2-3 lesions. For statistical reasons, we considered patients with 2-3 lesions to be in the same histological group.

High office systolic (SBP) and diastolic blood pressures (DBP) were defined as $\geq 140 \mathrm{~mm} \mathrm{Hg}$ and $90 \mathrm{~mm}$ $\mathrm{Hg}$, respectively, in two different measurements with an appropriate arm-cuff. Arterial hypertension was considered to be present when the patient had either high office SBP or DBP readings or was being treated with hypotensive drugs. Diabetes mellitus, hypercholesterolemia, and hypertriglyceridemia were defined as previously described [12]. Glomerular hyperfiltration was considered to be present when creatinine clearance was $>140 \mathrm{ml} / \mathrm{min}$. Microalbuminuria was defined as albumin levels between 30 and $300 \mathrm{mg}$ in 24-hour urine.

\section{Statistical Analysis}

To test for differences in proportions among patients according to the number of glomerular lesions, we used the Pearson's chi-square test, applying continuity correction or Fisher's exact test as appropriate. The Kruskal-Wallis test was used to compare medians of continuous renal variables among patient groups. To analyze changes between consecutive time points (baseline as well as 2, 5, 7 and 9 years), Mc Nemar's test and the Wilcoxon signed-rank test (paired tests) were used for binary and continuous variables, respectively. Linear mixed models were considered to assess the long-term 7-year effects of the number of obesity-related glomerular lesions on SBP, DBP, serum creatinine, creatinine clearance, and 24-hour albuminuria. The linear mixed models permitted the analysis of longitudinal data, taking into account the existing correlation between the repeated measures within each patient. All models were adjusted according to the patient's BMI evolution during follow-up. The regression coefficient of the time term (in years) in each of the linear mixed models was interpreted as the mean change per year of the corresponding renal variable. To assess differences between groups of patients according to the number of glomerular lesions, an interaction term was included in the model (number of lesions $\times$ BMI). A significant $p$ value of the regression coefficient of the interaction term meant different slopes (mean changes per year) in patients with a different number of glomerular lesions. Statistical analyses were made with the SAS 9.1 (SAS, Cary, NC, USA) package.

\section{Results}

General Data at Baseline, Short-Term Follow-Up, and Long-Term Follow-Up

Clinical, biochemical and histological data of all 92 morbidly obese patients at the time of renal biopsy (baseline) are shown in table 1 . Mean BMI was $53.9 \pm 9.1 \mathrm{~kg} / \mathrm{m}^{2}$. All patients had normal renal function tests.

When the 92 patients were subdivided according to the number of obesity-related glomerular lesions they presented, 17 had no lesion, 34 had 1 lesion (11 only podocyte hypertrophy, 6 only glomerulomegaly, and 17 only MMI and/or FSGS), 31 patients had 2 lesions (13 
Serra et al.: Long-Term Normal Renal Function after Drastic Weight Reduction in Patients with Obesity-Related Glomerulopathy

Table 1. Anthropometric, clinical, biochemical and histological description of data at the time of renal biopsy (baseline)

Total patients $(n=92)$

$\begin{array}{ll}\text { Female gender } & 58 \\ \text { Age, years } & 41.9 \pm 10(19-58)^{\mathrm{a}} \\ \text { Body weight, kg } & 148.5 \pm 25.6(96-220)^{\mathrm{a}} \\ \text { BMI, kg/m } & 53.9 \pm 9.1(40.3-80.9)^{\mathrm{a}} \\ \text { Obesity duration, months } & 238 \pm 105^{\mathrm{b}} \\ \text { Time of follow-up, months } & 76,3 \pm 41.6(6-144)^{\mathrm{a}} \\ \text { Smoker } & 47(51 \%)^{\mathrm{c}} \\ \text { High blood pressure } & 53(58 \%)^{\mathrm{c}} \\ \text { Diabetes mellitus } & 13(14 \%)^{\mathrm{c}} \\ \text { Impaired fasting glucose } & 20(22 \%)^{\mathrm{c}} \\ \text { Hypercholesterolemia } & 36(39 \%)^{\mathrm{c}} \\ \text { Hypertriglyceridemia } & 25(27 \%)^{\mathrm{c}} \\ \text { High glomerular filtration rate } & 30(33 \%)^{\mathrm{c}} \\ \text { Normal glomerular filtration rate } & 62(67 \%)^{\mathrm{c}} \\ \text { Without microalbuminuria } & \\ \text { (24-hour albuminuria }<30 \text { mg / } 24 \mathrm{~h}) & 50(55 \%)^{\mathrm{c}} \\ \text { Microalbuminuria } & \\ \text { (24-hour albuminuria } \geq 30 \text { and } \leq 300 \mathrm{mg} / 24 \mathrm{~h}) & 38(41 \%)^{\mathrm{c}} \\ \text { 24-hour albuminuria }>300 \text { and }<500 \mathrm{mg} / 24 \mathrm{~h} & 4(4 \%)^{\mathrm{c}} \\ \text { Treatment with ACEIs/ARBs } & 23(25 \%)^{\mathrm{c}} \\ \text { Obesity-related glomerulopathy } & \\ \quad \text { No lesions } & 17(18 \%)^{\mathrm{c}} \\ \quad \text { One lesion } & 34(37 \%)^{\mathrm{c}} \\ \quad \text { Two or three lesions } & 41(45 \%)^{\mathrm{c}}\end{array}$

${ }^{\mathrm{a}}$ Mean \pm SD and range. ${ }^{\mathrm{b}}$ Mean \pm SD. ${ }^{\mathrm{c}} \mathrm{n}(\%)$.

MMI and/or FSGS and podocyte hypertrophy, 16 MMI and/or FSGS and glomerulomegaly, and 2 glomerulomegaly and podocyte hypertrophy), and 10 patients had 3 lesions (MMI and/ or FSGS, glomerulomegaly, and podocyte hypertrophy).

The short- and long-term evolution of clinical, metabolic and renal function data of our cohort and of treatment with ACEIs and/or ARBs drugs are shown in table 2. Mean BMI and creatinine clearance decreased significantly during the first 2 years of follow-up, increased between 2 and 5 years after surgery, and remained stable thereafter. Mean SBP and DBP fell significantly during the first 2 years after bariatric surgery and remained stable thereafter. Mean serum creatinine and 24-hour albuminuria decreased throughout the first 7 years of follow-up. All metabolic indicators improved significantly during the first 2 years postsurgery. Improvement in HDL-cholesterol levels persisted for 5 years following surgery. Increases in serum total cholesterol, glucose and HbA1c were observed in the long-term follow-up.

No short- or long-term differences in relation to serum creatinine and creatinine clearance were observed between patients who presented with basal 24-hour albuminuria $\geq 30 \mathrm{mg} /$ $24 \mathrm{~h}$ or with basal 24-hour albuminuria $<30 \mathrm{mg} / 24 \mathrm{~h}$.

Differences in Anthropometric and Renal Function Variables at Various Follow-Up Points

(Baseline as well as 2, 5, 7, and 9 Years) according to the Number of Obesity-Related Glomerular Lesions (fig. 1)

At baseline, the three groups of patients (no lesions, 1 lesion, and 2-3 lesions) presented significant differences in BMI ( $p<0.05)$, but not in SBP and DBP, serum creatinine, creatinine 


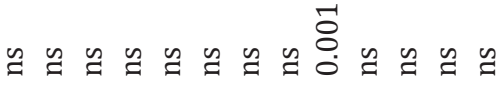

Serra et al.: Long-Term Normal Renal Function after Drastic Weight Reduction in Patients with Obesity-Related Glomerulopathy
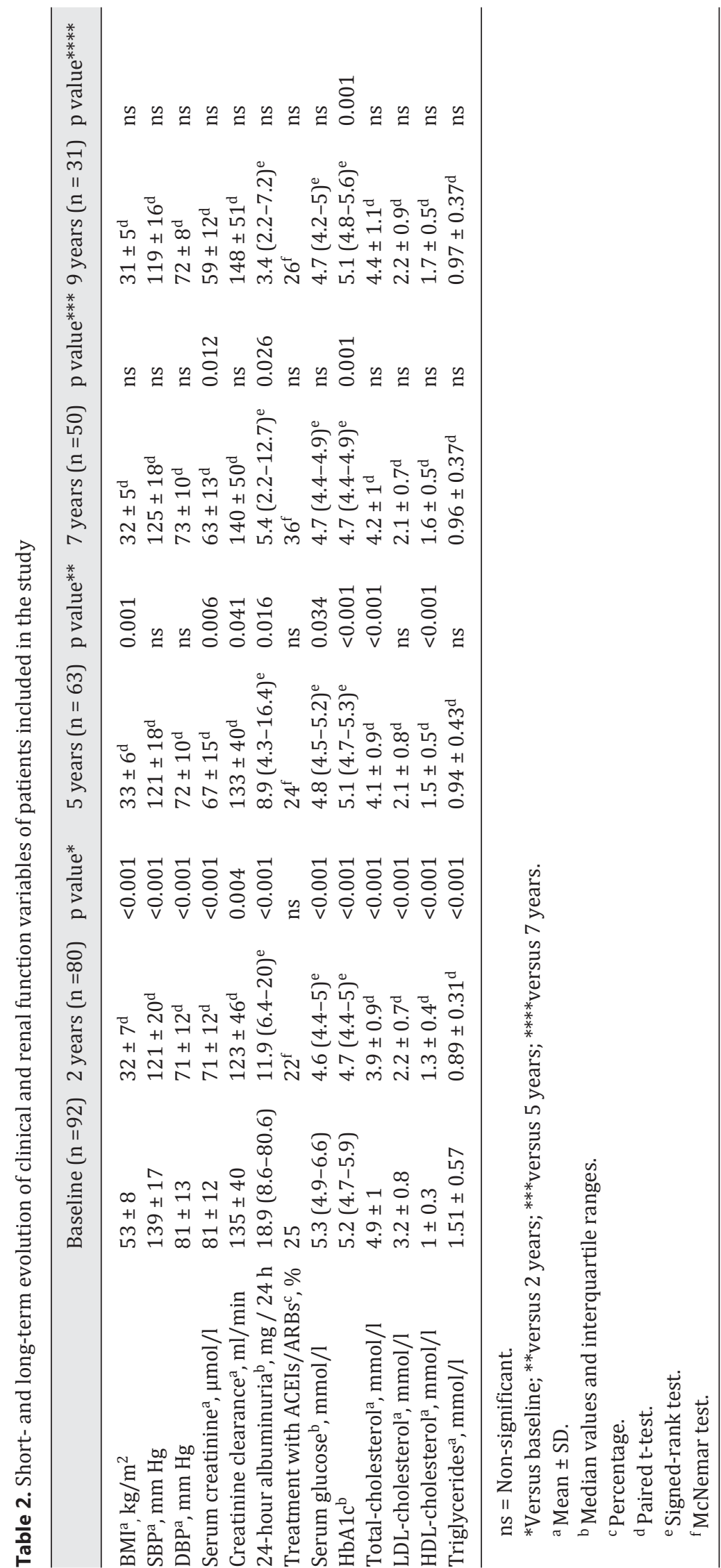
Fig. 1. a Serum creatinine, b creatinine clearance, c 24-hour albuminuria and $\mathbf{d}$ BMI at baseline, and at 2, 5, 7 and 9 years after bariatric surgery in patients with a different number of obesity-related glomerular lesions (no lesion, 1 lesion and 2-3 lesions).

(a) $p$ value: Kruskal-Wallis test between groups.

${ }^{(1)} p$ value: signed-rank test, paired data within groups.
Serra et al.: Long-Term Normal Renal Function after Drastic Weight Reduction in Patients with Obesity-Related Glomerulopathy

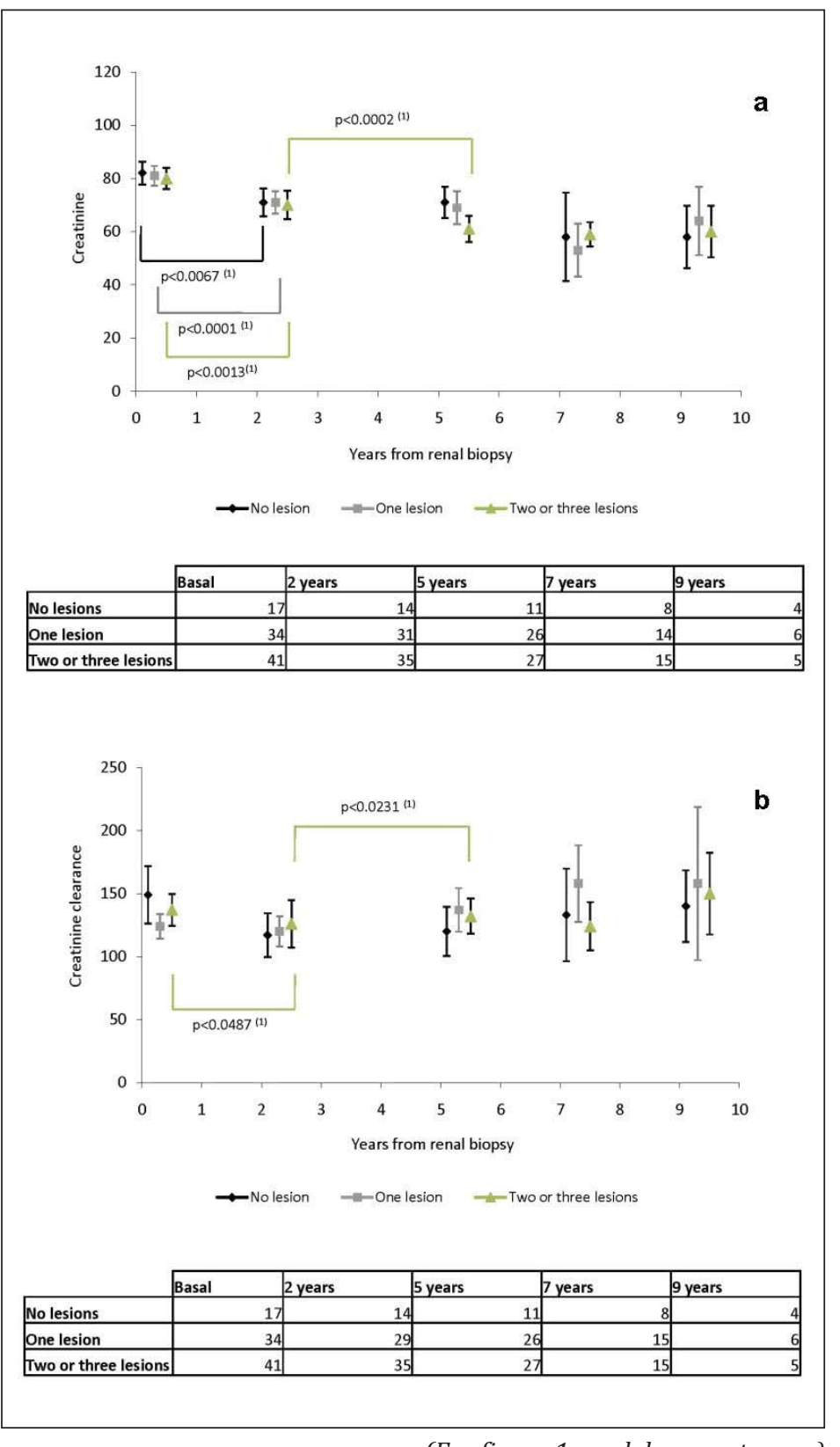

(For figure $1 c$ and d see next page.)

clearance and 24-hour albuminuria. Patients with glomerular lesions had higher BMI than patients without glomerular lesions.

No differences were found among the 3 groups with regard to SBP and DBP, serum creatinine, creatinine clearance and 24-hour albuminuria at 2, 5 and 9 years after bariatric surgery. Differences were observed at 7 years post-surgery for SBP $(p<0.05)$ and DBP $(p<0.05)$, but not in renal function parameters or 24-hour albuminuria.

Differences in Anthropometric and Renal Function Variables during Follow-Up according to the Number of Obesity-Related Glomerular Lesions (fig. 1).

Long-term follow-up of BMI, serum creatinine, creatinine clearance, and 24-hour albuminuria in patients with no lesions, 1 lesion and 2-3 lesions of obesity-related glomeru- 
Serra et al.: Long-Term Normal Renal Function after Drastic Weight Reduction in Patients with Obesity-Related Glomerulopathy

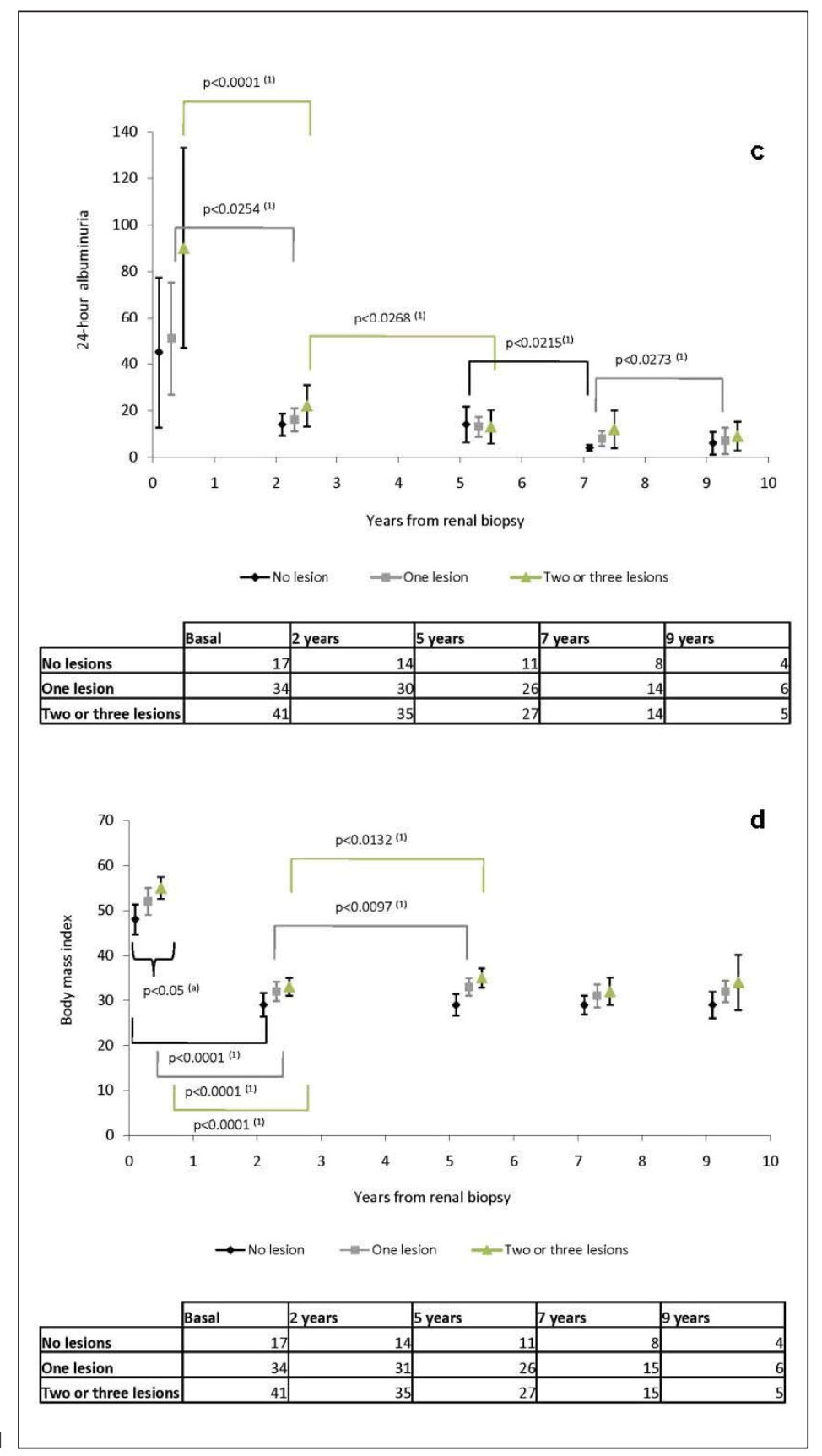

lopathy showed a significant BMI decrease during the first 2 years after bariatric surgery in all 3 groups. BMI increased slightly between 2 and 5 years after surgery in patients with 1 lesion and in patients with 2-3 lesions.

Serum creatinine dropped significantly in the 3 groups during the early follow-up phase and continued to decrease between 2 and 5 years post-surgery in patients with 2 or 3 lesions of obesity-related glomerulopathy.

Creatinine clearance fell significantly early after bariatric surgery and rose between 2 and 5 years after surgery in patients with 2 or 3 lesions of obesity-related glomerulopathy; however, no changes were observed in the other histological groups. 

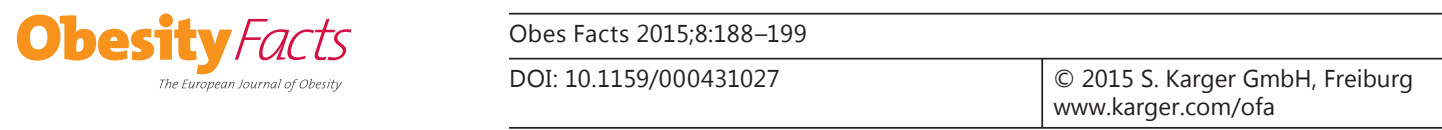

Serra et al.: Long-Term Normal Renal Function after Drastic Weight Reduction in Patients with Obesity-Related Glomerulopathy

Table 3. Relationship between the number of obesity-related glomerular lesions and clinical and renal function variables after 7 years of follow-up (longitudinal linear mixed models ${ }^{a}$ )

\begin{tabular}{|c|c|c|c|c|c|c|c|}
\hline & \multicolumn{2}{|l|}{ Time, years } & \multicolumn{2}{|l|}{ BMI } & \multicolumn{2}{|c|}{ Number of lesions } & \multirow{2}{*}{$\begin{array}{l}\mathrm{p} \text { value } \\
\text { number of } \\
\text { lesions } \times \mathrm{BMI}^{\mathrm{l}}\end{array}$} \\
\hline & slope (SE) & $\mathrm{p}$ value & slope (SE) & $\mathrm{p}$ value & slope (SE) & $\mathrm{p}$ value & \\
\hline SBP & $-0.02(0.04)$ & 0.1920 & $1.05(0.15)$ & $<0.0001$ & $9.5(3.7)$ & 0.0126 & 0.0165 \\
\hline DBP & $-0.02(0.03)$ & 0.4788 & $0.49(0.12)$ & $<0.0001$ & $5.0(3.0)$ & 0.0959 & 0.1957 \\
\hline Serum creatinine & $-0.08(0.03)$ & 0.0008 & $0.31(0.11)$ & 0.0046 & $-3.4(2.8)$ & 0.2312 & 0.6063 \\
\hline Creatinine clearance & $0.30(0.08)$ & 0.0003 & $0.949(0.35)$ & 0.0076 & $-0.4(8.9)$ & 0.9678 & 0.6753 \\
\hline 24-hour albuminuria ${ }^{c}$ & $-0.004(0.001)$ & $<0.0001$ & $0.007(0.004)$ & 0.0570 & $-0.15(0.1)$ & 0.1333 & 0.0614 \\
\hline
\end{tabular}

${ }^{\mathrm{a}}$ Linear mixed models: outcome $=$ intercept + year $+\mathrm{BMI}+$ number of lesions + number of lesions $\times$ BMI.

${ }^{\mathrm{b}} \mathrm{P}$ value of the interaction term number of lesions $\times \mathrm{BMI}$ of the adjusted linear mixed model.

${ }^{\mathrm{c}}$ The variable is transformed as $\log 10$.

24-hour albuminuria decreased significantly during early follow-up in patients with 1 or more lesions of obesity-related glomerulopathy. A significant 24-hour albuminuria drop was seen between 2 and 5 years of follow-up in patients with 2 or 3 glomerular lesions, between 5 and 7 years after surgery in patients with no lesions, and between 7 and 9 years in patients with 1 lesion.

\section{Long-Term Effects of Obesity-Related Glomerular Lesions on Renal Function Variables}

(table 3).

The mixed models over a 7-year follow-up period showed the number of obesity-related glomerular lesions to be associated with a SBP increase, but not with changes in the other variables analyzed (DBP, serum creatinine, creatinine clearance, and 24-hour albuminuria).

Time and BMI significantly influenced the evolution of renal function and 24-hour albuminuria. On the other hand, BMI significantly contributed to the evolution of SBP and DBP.

Similar results were obtained when the BMI-adjusted influence of the different numbers of obesity-related glomerular lesions on the evolution of blood pressure, renal function, and 24-hour albuminuria was analyzed over a 9-year follow-up period.

\section{Discussion}

This is the first prospective observational study describing the long-term evolution of blood pressure and renal function variables in a bariatric surgery-treated cohort of 92 morbidly obese patients with pre-surgical evidence of obesity-related glomerulopathy, normal renal function, and post-surgical weight loss.

More specifically, this study is the first to analyze the influence of the number of obesityrelated glomerular lesions on the evolution of blood pressure, renal function, and microalbuminuria after adjustment for confounding variables such as BMI changes.

Recently, several studies addressed the short-term evolution of renal function and proteinuria in obese patients after weight loss [2, 3, 8, 9, 19, 24-27], and the results have been summarized in several meta-analyses [4,5]. Improvements in renal function, proteinuria, and albuminuria were the most common findings and occurred in patients with or without established chronic renal failure $[5,18,28,29]$. These improvements were more pronounced in bariatric surgery-treated patients than in those whose weight loss was induced by lifestyle methods. This difference was probably due to the easier maintenance of weight loss in 
Serra et al.: Long-Term Normal Renal Function after Drastic Weight Reduction in Patients with Obesity-Related Glomerulopathy

bariatric surgery-treated patients. Follow-up periods oscillated between 6 months and 2 years in the majority of publications, and studies with more prolonged follow-up periods are lacking. Therefore, no knowledge exists regarding the long-term renal function evolution in morbidly obese patients undergoing drastic weight loss after bariatric surgery, either in cases with initially normal or abnormal renal function and with or without albuminuria [5].

We studied long-term renal function evolution after bariatric surgery (mean follow-up > 6 years) in 92 morbidly obese patients with basal normal renal function without albuminuria or with low-range albuminuria. In our work, blood pressure remained within normal limits 9 years after surgery (with or without antihypertensive treatment), serum creatinine and albuminuria decreased over time, and creatinine clearance fell in the first 2 years of followup and rose afterwards in some cases. These long-range discordant results between serum creatinine and creatinine clearance could suggest that serum creatinine might not be an accurate method for evaluating renal function in patients losing weight. Other factors, such as muscle mass loss, could influence creatinine levels more than other renal function variables.

Several factors such as $\mathrm{NaCl}$ management, changes in diet type, or food tolerance could explain the creatinine clearance decrease observed during the first 2 years post bariatric surgery and its later increase. This long-term rise in creatinine clearance was accompanied by a slight increase in other variables such as serum HbA1c, total cholesterol, HDL- and LDLcholesterol, and triglyceride levels [30]. As reported in other series of obese or non-obese patients, long-term studies of the effects of different diet types on slimmed-down morbidly obese patients could help to explain these changes [31].

Since in clinical practice the presence of microalbuminuria is considered a major marker for renal damage, we studied whether the presence of basal albuminuria could influence the long-term renal function prognosis and found no long-term differences in relation to renal function parameters. The early intervention in these patients before they presented proteinuria might have avoided a worse renal prognosis. No control studies on this subject are available.

The prognosis of renal function in obese patients with obesity-related glomerulopathy is very poorly known. An unfavorable spontaneous evolution of renal function has been described in obese patients with proteinuria and/or renal failure and glomerulomegaly and/or FSGS [11, 16]. $15 \%$ of these patients had doubled their serum creatinine levels or developed end-stage renal disease after 5 years, whereas $45 \%$ of them had doubled their serum creatinine levels or developed end-stage renal disease after 8 years [11]. In those studies, the risk of progression to renal failure correlated with serum creatinine levels at the time of diagnosis [16].

Little is known on the short- and long-term renal function changes in obese patients with obesity-related glomerulopathy undergoing drastic weight reduction. Only a few studies on this type of patient with proteinuria and/or abnormal renal function and glomerulomegaly and/or FSGS lesions have been published $[18,19,32]$. Slowing of renal function deterioration and decreased proteinuria have been reported in these patients after drastic weight loss [18, $19,32]$. However, there are no short- or long-term prospective studies of renal function evolution in bariatric surgery-treated morbidly obese patients with pre-surgical evidence of normal renal function and obesity-related glomerular lesions and postsurgical drastic weight loss.

In our bariatric surgery-treated morbidly obese patients with initially normal renal function and different types and number of obesity-related glomerular lesions, the long-term prognosis was good, regardless of the type of glomerular lesion and the number of glomerular lesions observed on renal biopsy. These good results differ from those obtained from the historic obese controls with no bariatric surgery $[11,16]$. Blood pressure, renal function, and urine albumin levels remained within normal limits throughout the long-term follow-up 
Serra et al.: Long-Term Normal Renal Function after Drastic Weight Reduction in Patients with Obesity-Related Glomerulopathy

period, irrespective of the presence or absence of glomerular lesions. Thus, we can assume that drastic weight loss exerted a positive influence on the renal prognosis of these patients. For ethical reasons, our study did not include a control group of morbidly obese patients in spontaneous evolution. Similarly, after adjustment for BMI changes, longitudinal linear mixed model analysis demonstrated that the number of obesity-related glomerular lesions did not influence the evolution of serum creatinine levels or creatinine clearance. Likewise, the evolution of SBP would have been influenced by the number of obesity-related glomerular lesions. No similar longitudinal linear mixed model studies of renal function variables in morbidly obese patients with obesity-related glomerulopathy exist for comparison with our results.

The main strength of this long-term observational study lies in the fact that it included a large cohort (92 patients) of bariatric surgery-treated morbidly obese patients with renal biopsy before drastic weight loss. Surgical techniques used in recent years prevent renal biopsies being taken during bariatric surgery which renders our study difficult to repeat.

We acknowledge several limitations of our ongoing observational study. For example, subjects were mainly females, and some patients were treated with ACEIs and/or ARBs, drugs that may affect the evaluation of albuminuria (however, the percentage of patients treated with these drugs was similar at the beginning and the end of follow-up). Our study (of patients selected on the basis of surgical indications) is the only one addressing the long-term renal function evolution of patients with normal renal function without albuminuria or with lowdegree albuminuria at baseline. Serum creatinine levels and 24-hour creatinine clearance were used to evaluate renal function. GFR was estimated using creatinine clearance measured in a 24-hour urine sample without correction for body surface area, since correction would considerably underestimate the real value of GFR [33].

\section{Conclusions}

Drastic weight loss in bariatric surgery-treated morbidly obese patients with initially normal renal function results in an improvement of arterial hypertension and albuminuria levels and in a short- and long-term maintenance of normal renal function variables.

Long-term evolution of renal function and albuminuria was not influenced by the number of obesity-related glomerular lesions. The number of glomerular lesions would have negatively affected the long-term course of SBP.

Thus, it is reasonable to assume that drastic weight loss and improvement in variables such as arterial hypertension contribute to the excellent renal prognosis of morbidly obese patients with certain obesity-related glomerular lesions but free of other kidney conditions.

\section{Ethics}

Patients were selected for bariatric surgery following the criteria of our hospital's Obesity Unit. Renal biopsies were obtained when possible, with a maximum of two punctures in each patient. The project was approved by the hospital Institutional Review Board (protocol No. E0-00-010). All patients gave their written informed consent to participate in the study.

\section{Acknowledgements}

We wish to thank Miss Christine O'Hara for her help with manuscript editing. This work was supported in part by a grant from the Autonomous University of Barcelona. 
Serra et al.: Long-Term Normal Renal Function after Drastic Weight Reduction in Patients with Obesity-Related Glomerulopathy

\section{Disclosure Statement}

Absence of any interest to disclose.

\section{References}

1 Hsu CY, Mc Culloch CE, Iribarren C, Darbinian J, Go AS: Body mass index and risk for end-stage renal disease. Ann Intern Med 2006;144:21-28.

2 Serra A, Granada ML, Romero R, Bayés B, Cantón A, Bonet J, Rull M, Alastrue A, Formiguera X: The effect of bariatric surgery on adipocytokines, renal parameters and other cardiovascular risk factors in severe and very severe obesity: 1-year follow-up. Clin Nutr 2006;25:400-408.

-3 Navarro-Díaz M, Serra A, Romero R, Bonet J, Bayés B, Homs M, Pérez N, Bonal J: Effect of drastic weight loss after bariatric surgery on renal parameters in extremely obese patients: long-term follow-up. J Am Soc Nephrol 2006;17(12 suppl 3):S213-S217.

4 Afshinnia F, Wilt TJ, Duval S, Esmaeili A, Ibrahim HN: Weight loss and proteinuria: systematic review of clinical trials and comparative cohorts. Nephrol Dial Transplant 2010;25:1173-1183.

-5 Navaneethan SD, Yehnert H, Moustarah F, Schreiber MJ, Schauer PR, Beddhu S: Weight loss interventions in chronic kidney disease: a systematic review and meta-analysis. Clin J Am Soc Nephrol 2009;4:1565-1574.

6 Saiki A, Nagayama D, Ohhira M, Endoh K, Ohtsuka M, Koide N, Oyama T, Miyashita Y, Shirai K: Effect of weight loss using formula diet on renal function in obese patients with diabetic nephropathy. Int J Obes 2005;29: 1115-1120.

7 Morales E, Valero MA, Leon M, Hernández E, Praga M: Beneficial effects of weight loss in overweight patients with chronic proteinuric nephropathies. Am J Kidney Dis 2003;41:319-327.

-8 Alexander JW Goodman HR, Martin Hawve LR, Cardi MA: Improvement and stabilization of chronic kidney disease after gastric bypass. Surg Obes Relat Dis 2009;5:237-241.

-9 Navaneethan SD, Yehnert H: Bariatric surgery and progression of chronic kidney disease. Surg Obes Relat Dis 2009;5:662-665.

10 Weisinger JR, Kempson RL, Eldridge FL, Swenson RS: The nephrotic syndrome: a complication of massive obesity. Ann Intern Med 1974;81:440-447.

11 Kambham N, Markowitz GS, Veleri AM, Lin J, D’Agati VD: Obesity-related glomerulopathy: an emerging epidemic. Kidney Int 2001;59:1498-1509.

$\checkmark 12$ Serra A, Romero R, López D, Navarro M, Esteve A, Perez N, Alastrue A, Ariza A: Renal injury in the extremely obese patients with normal renal function. Kidney Int 2008;73:947-955.

$\checkmark 13$ Chen HM, Li SJ, Chen HP, Wang QW, Li LS, Liu ZH: Obesity-related glomerulopathy in China: a case series of 90 patients. Am J Kidney Dis 2008;52:58-65.

14 Goumenos DS, Kawar B, El Nahas M, Conti S, Wagner B, Spyropoulos C, Vlachojannis JG, Benigni A, Kalfarentzos F: Early histological changes in the kidney of people with morbid obesity. Nephrol Dial Transplant 2009;24: 3732-3738.

15 Adelman RD, Restaino IG, Alon US, Blowey DL: Proteinuria and focal segmental glomerulosclerosis in severely obese adolescents. J Pediatr 2001;138:481-485.

-16 Praga M, Hernández E, Morales E, Pérez Campos A, Valero MA, Martínez MA, León M: Clinical features and long-term outcome of obesity-associated focal segmental glomerulosclerosis. Nephrol Dial Transplant 2001; $16: 1790-1798$

17 Georgaki Angelaki H, Stergiou N, Manolaki N, Nakopoulou L, Syriopoulou VP, Roma-Giannikou E: Histological deterioration of obesity-related glomerulopathy despite the loss of proteinuria with weight reduction. Pediatr Nephrol 2010;25:1573-1574.

-18 Huan Y, Tomaszewski JE, Cohen DL: Resolution of nephrotic syndrome after successful bariatric surgery in patient with biopsy-proven FSGS. Clinical Nephrol 2009;71:69-73.

19 Fowler SM, Kon V, Ma L, Richards WO, Fogo AB, Hunley TE: Obesity-related focal and segmental glomerulosclerosis: normalization of proteinuria in an adolescent after bariatric surgery. Pediatr Nephrol 2009;24: 851-855.

20 Shen WW, Chen HM, Chen H, Xu F, Li LS, Liu ZH: Obesity-related glomerulopathy: body mass index and proteinuria. Clin J Am Soc Nephrol 2010;5:1401-1409.

21 Consensus Development Conference Statement: Gastrointestinal surgery for severe obesity. Ann Intern Med 1991;115:794-798.

22 Fobi MA, Lee H, Holness R, Cabinda D: Gastric bypass operation for obesity. World J Surg 1998;22:925-935.

-23 Salmon PA: Gastroplasty with distal gastric bypass: a new and more successful weight loss operation for the morbidly obese. Can J Surg 1988;31:111-113.

24 Agrawal V, Khan I, Rai B, Krause KR, Chengelis DL, Zalesin KC, Rocher LL, McCullough PA: The effect of weight loss after bariatric surgery on albuminuria. Clin Nephrol 2008;70:194-202.

25 Chagnac A, Weinstein T, Herman M, Hirsh J, Gafter U, Ori Y: The effects of weight loss on renal function in patients with severe obesity. J Am Soc Nephrol 2003;14:1480-1486. 
26 Agnani S, Vachharajani VT, Gupta R, Atray NK, Vachharajani TJ: Does treating obesity stabilize chronic kidney disease? BMC Nephrol 2005;6:7.

27 Soto FC, Higa-Sansone G, Copley JB, Berho M, Kennedy C, LoMenzo E, Podkameni D, Szomstein S, Rosenthal RJ: Renal failure, glomerulonephritis and morbid obesity: improvement after rapid weight loss following laparoscopic gastric bypass. Obes Surg 2005;15:137-140. Currie A, Chetwood A, Ahmed AR: Bariatric surgery and renal function. Obes Surg 2011;21:528-539. Schuster DP, Teodorescu M, Mikami D, Foreman K, Rogers P, Needleman BJ: Effect of bariatric surgery on normal and abnormal renal function. Surg Obes Relat Dis 2011;7:459-464.

-30 Ritz E, Koleganova N: Obesity and chronic kidney disease. Semin Nephrol 2009;29:504-511.

-31 Friedman AN, Ogden LG, Foster GR, Klein S, Stein R, Miller B, Hill JO, Brill C, Bailer B, Rosembaum DR, Wyatt HR: Comparative effects of low-carbohydrate high-protein versus low-fat diets on the kidney. Clin J Am Soc Nephrol 2012;7:1103-1111.

32 Ramírez J, Carpio D, Mezzano S, Mukdsi J, Ardiles L: Cirugía bariátrica en pacientes con glomeruloesclerosis focal y segmentaria secundaria a obesidad. Nefrologia 2009;29:266-269.

33 Delanaye P, Radermecker RP, Rorive M, Depas G, Krzesinski JM: Indexing glomerular filtration rate for body surface area in obese patients is misleading: Concept and example. Nephrol Dial Transplant 2005;20:20242028. 